load in each tract. A negative binomial regression model was used to test the association between violent crime events and CVL, including spatial lag from shared-boundary census tracts as well as mean age, proportion male, labor force participation, educational attainment, and residential instability as key covariates.

Results The annual violent crime rate in Baltimore City in the 5-year period from 2012-2016 was 29.5 per 1,000 population; rates varied widely between census tracts, ranging from 2 to 189.4 per 1,000 . The mean CVL was 4.27 per 1,000 population, with a range from 0 to 18.8 by census tract. In the adjusted model, a 100-unit increase in violent crimes was associated with a 19\% increase in CVL (RR: 1.19, 95\% CI: $1.13,1.27)$.

Conclusion Our study shows a statistically significant association between violent crime rates and HIV transmission risk in local areas. This study highlights the need for community-level interventions aimed to address effects of violence exposure in order to effectively combat the ongoing HIV epidemic among vulnerable populations in urban settings.

Disclosure No significant relationships.

\section{P302 ACHIEVING THE THIRD 90: KEEPING PREGNANT AND BREASTFEEDING WOMEN LIVING WITH HIV VIRALLY SUPPRESSED IN WESTERN NIGERIA}

${ }^{1}$ Saheed Usman, ${ }^{2}$ biwumi Usman*. ${ }^{1}$ APIN Public Health Intiatives, Abuja, Nigeria; ${ }^{2}$ Kids and Teens Resource Centre, Akure, Nigeria

\subsection{6/sextrans-2019-sti.416}

Background In 2016, Nigeria transitioned to 'Test \& Treat', a policy where all people living with HIV (PLHIV) are treated with lifelong antiretroviral therapy (ART) regardless of clinical or immunological status. Hypothesis tested was antiretroviral therapy adherence effect on viral load outcome. The aim of this study is to assess the viral load suppression rates among HIV positive pregnant \& breastfeeding women in Western Nigeria.

Methods This study was an observational prospective study of pregnant \& breastfeeding HIV positive subjects already initiated on antiretroviral therapy for at least six months, enrolled in health facilities across supported facilities in Western Nigeria, during a 12-month observation period starting October 2016 till September 2017. Quantitative viral load analysis was done using Polymerase Chain Reaction, Roche Cobas Taqman 96 Analyzer.

Results A total of 243 subjects were recruited. The mean age of $32.58 \pm 4.18$ years. 69 are breastfeeding while 174 were pregnant women. 208 (85.6\%) \& 188 (77.4\%) had viral suppression of $<1000 \&<50$ RNA copies per ml respectively. The 35 subjects went through peer counseling by trained PLHIV and enhanced adherence counseling (EAC) for three months and viral load test repeated three further months after, which made 221 (90.9\%) \& 202 (83.1\%) of the subjects have $<1000$ RNA \& $<50$ RNA copies per ml respectively during the observation. The PLHIVs joined the institutionalized social-media driven support group \& decentralized care model ensuring they achieve the third 90 at undetectable level. ART adherence has significant effect on viral load outcome $\left(\chi^{2}=5.86\right.$, df $\left.=1, \mathrm{P}=0.001\right)$.

Conclusion ART adherence counseling is key to the achieving viral suppression and determine infection prognosis, thus, developing robust continuous quality improvement (CQI) plans to address issues across the cascade ultimately helping in the monitoring of HIV/AIDS disease progression and decrease treatment failure tendencies.

Disclosure No significant relationships.

\section{P304 ACHIEVING THE THIRD 90: KEEPING ADOLESCENTS LIVING WITH HIV VIRALLY SUPPRESSED IN RURAL NIGERIA IN THE ERA OF TEST AND TREAT}

Saheed Usman*, Prosper Okonkwo, Oluwatoyin Jolayemi, Jay Osi-Samuels, Patrick Akande, Babatunde Ladi-Akinyemi, Oluremi Olaitan, Femi Owolagba, Matthias Alagi, Eke Ofuche. APIN Public Health Intiatives, Abuja, Nigeria

\subsection{6/sextrans-2019-sti.417}

Background In 2016, Nigeria transitioned to 'Test \& Treat', a policy where all people living with HIV (PLHIV) are treated with lifelong antiretroviral therapy (ART). There are unique challenges achieving viral suppression in ALHIV mainly due to increased stigma \& lack of social support. Hypothesis tested was ART adherence effect on viral load outcome. We examined viral suppression among adolescents living with HIV in Western Nigeria.

Methods This study was an observational prospective cohort study of adolescents living with HIV (ALHIV) already initiated on antiretroviral therapy for at least six months, enrolled in health facilities across supported facilities in Western Nigeria, during a 12-month observation period starting October 2016 till September 2017. Quantitative viral load analysis was done using Polymerase Chain Reaction, Roche Cobas Taqman 96 Analyzer.

Results A total of 126 (64 males \& 62 females) subjects were recruited. The mean age of $13.58 \pm 4.26$ years. $83(65.9 \%)$ $\& 71(56.3 \%)$ had viral suppression of $<1000 \&<50$ RNA copies per $\mathrm{ml}$ respectively. The 43 subjects went through peer counseling by trained ALHIV and enhanced adherence counseling (EAC) for three months and viral load test repeated three further months after, which made 113 (89.7\%) \& 101 (80.1\%) of the subjects have $<1000$ RNA $\&<50$ RNA copies per $\mathrm{ml}$ respectively during the observation. The ALHIVs joined the institutionalized social-media driven support group $\&$ adolescent decentralized care model ensuring they achieve the third 90 at an undetectable level. ART adherence has significant effect on viral load outcome $\left(\chi^{2}=6.42\right.$, df $=1, \mathrm{P}$ $=0.001)$.

Conclusion ART adherence counseling is key to the achieving viral suppression and determine infection prognosis, thus, developing robust continuous quality improvement (CQI) plans to address issues across the cascade ultimately helping in the monitoring of HIV/AIDS disease progression and decrease treatment failure tendencies.

Disclosure No significant relationships. 